\title{
EFFECT OF POWDER CLEANLINESS ON THE FATIGUE BEHAVIOR OF POWDER METALLURGY NI-DISK ALLOY UDIMET 720
}

\author{
P. Kantzos ${ }^{1}$, P. Bonacuse ${ }^{2}$, J. Telesman ${ }^{3}$, T. Gabb ${ }^{3}$, R. Barrie ${ }^{2}$ and A. Banik ${ }^{4}$ \\ ${ }^{1}$ Ohio Aerospace Institute, Cleveland $\mathrm{OH}$ \\ ${ }^{2}$ Army Research Laboratory, Cleveland, $\mathrm{OH}$ \\ ${ }^{3}$ NASA Glenn Research Center, Cleveland, $\mathrm{OH}$ \\ ${ }^{4}$ Special Metals Corporation, Princeton, KY
}

Keywords: Superalloy, powder metallurgy, low cycle fatigue, inclusions

\begin{abstract}
Non-metallic inclusions, which are inherent to the powder metallurgy process, can have a deleterious effect on the fatigue life of turbine disks. An artificial seeding study, where both the size and occurrence rate of the ceramic seeds were well controlled and characterized, was employed as a means of studying the effects of powder dirtiness on fatigue life. Various methods of quantifying cleanliness; quantitative metallography, quantitative fractography, and heavy liquid separation, were evaluated and compared. In addition, extensive metallographic characterization of seeded extrusions and forgings was performed in order to quantify the effect of mechanical processing on inclusion morphology.
\end{abstract}

\section{Introduction and Background}

Powder metallurgy (P/M) nickel base superalloys are presently utilized in turbine disks in many major commercial and military engines. The $\mathrm{P} / \mathrm{M}$ process in many cases is the only way to produce the highly alloyed chemistries capable of providing the mix of mechanical and thermal properties required in the turbine disk operating environment.

Non-metallic inclusions are inherent in the $\mathrm{P} / \mathrm{M}$ process, much like they are a by-product of most other metallurgical process where melting occurs in refractory crucibles. The presence of large inclusions, especially on the surface, has long been recognized as deleterious to the fatigue behavior of structural alloys [1]. Under certain loading conditions inclusions act as premature crack initiation sites and can result in catastrophic failure. Furthermore, the failure of a turbine disk cannot be contained resulting in loss of the engine and perhaps the aircraft. Historically, the mitigation of catastrophic failures from inclusions has been achieved through the continuous improvements in powder processing and handling by powder manufacturers as well as the trend towards the use of finer powders and mechanical processing by engine manufacturers. Modern powder facilities can produce fine powder $(<-270$ mesh $)$ with inclusion contamination levels less than $1 \mathrm{ppm}$ by weight. Nonetheless, in lifing of disk components the presence of large inclusions, albeit rare, remains a concern due to the large volume of highly stressed material and the criticality of failure. It is obvious that lifing a component by assuming the worst possible flaw in the worst location is not only overly conservative, it doesn't provide a real means for taking advantage of improvement in powder quality, and processing. The challenges in lifing disks, where failure from an inclusion is a possible failure mode, rest on the ability to quantify powder cleanliness and correlate it to the fatigue behavior of the material. As powder cleanliness improves, traditional methods of screening material through fatigue testing is not cost effective or efficient because large volumes of material will need to be tested in order to statistically quantify the inclusion initiation life debit. Similar problems plague other test methods such as spin pit testing, where although large volumes can be tested performing more than a few experiments would be cost prohibitive. Such methods would also not be cost effective in evaluating production process variability.

Probabilistic lifing methods provide a means to life a component or fleet of components, where rare and random inclusions can be present, with a known reliability. In order to take advantage of probabilistic lifing it is imperative to quantify powder cleanliness and it effect on fatigue life.

In this report, an artificial seeding study was employed as a mean of addressing the issues of: 1) quantifying powder cleanliness, 2) the effect of processing on inclusions, and 3) the consequence of inclusions on fatigue life. The use of a seeding study not only provides the opportunity to evaluate wellcontrolled and statistically characterized material but also alleviates the problem of testing a prohibitive large number of specimens.

In this study, extensive quantitative metallography, quantitative fractography, and the heavy liquid separation process (HLS) were all used to characterize both seeded and unseeded Udimet 720. Fatigue testing was performed to quantify the effect of inclusions on fatigue life.

\section{Procedures}

Material Processing and Seeding Parameters

The seeding study was performed on $\mathrm{P} / \mathrm{M}$ alloy Udimet 720 (U720) which was atomized in argon and sieved to -270 mesh by Special Metals Corporation using state-of-the-art full-scale production facilities and practices to insure the cleanest possible powder [2].

Table I. P/M alloy Udimet 720 Chemistry (w/o)

\begin{tabular}{|c|c|c|c|c|c|c|c|}
\hline $\mathrm{Cr}$ & $\mathrm{Co}$ & $\mathrm{Mo}$ & $\mathrm{W}$ & $\mathrm{Ti}$ & $\mathrm{Al}$ & $\mathrm{Ni}$ & Minor \\
\hline 16.2 & 14.7 & 3.1 & 1.3 & 5.1 & 2.5 & $\mathrm{Bal}$ & $\mathrm{C}, \mathrm{B}, \mathrm{Zr}$ \\
\hline
\end{tabular}

The powder was separated into three lots: two lots were seeded with alumina particles; the other was consolidated in the unseeded condition for comparison.

Two different types of alumina seeds were used: Ram90 sieved at $-270+325$ mesh and AlcoaT64 at $-140+170$ mesh (Figure 1). The Ram90 represented a typical friable Type II inclusion at a size distribution typical of production disk alloys. While 
AlcoaT64 represented a hard blocky Type I inclusion at a size distribution that could occur in a possible contamination event (such as a broken screen) and represents the upper limit of defects expected in production powder. Relatively high seeding rates of 325 seeds $/ \mathrm{cm}^{3}$ for Ram90 and 70 seeds $/ \mathrm{cm}^{3}$ AlcoaT64 were chosen to result in approximately 10 seed intersections per fatigue bar surface to ensure surface initiation failures.


Figure 1. Alumina particles used in the seeding study: a)Ram90 sieved at $-270+325$ and b)AlcoaT64 sieved at $140+170$.

Before blending the seeds with the powder, their size distribution was determined using image analysis on photographs of loose seeds. The seed size distributions were typically log-normal. The average seed diameters were approximately $54 \mu \mathrm{m}$ and $122 \mu \mathrm{m}$ for the $-270+325 \mathrm{Ram} 90$ and the AlcoaT64 -140+170 seeds respectively. The seeds were added to the powder by weight which was determined by counting and weighing several batches of loose seeds.

Both seeded and unseeded powder was consolidated using typical disk alloy processing [3]: hot isostatic pressing followed by $6: 1$ extrusion, and then isothermally forged into pancakes about $17.5 \mathrm{~cm}$ dia. by $4 \mathrm{~cm}$ thick. All forgings were given a subsolvus solution heat treatment, followed by an oil quench and aging treatment typical for U720 [4]. The resulting microstructure, grain size ASTM 11, as well as the primary and cooling $\gamma$ ' structure was consistent for all the forgings.

\section{Mechanical Testing}

Specimen blanks were machined from the pancake forgings with a circumferential (hoop) orientation. Testing was performed using a large cylindrical fatigue bar with a $10.2 \mathrm{~mm}$ diameter and $31.8 \mathrm{~mm}$ long gage section. Strain control fatigue tests were performed at $650 \mathrm{C}$ in a closed loop servo-hydraulic testing machine at strain ranges between $1.2 \%$ to $0.6 \%$ and R-ratios of $1,0.0$, and 0.5 .

Prior to testing, some of the fatigue bar surfaces were examined in the scanning electron microscope (SEM) via a specially designed stage which systematically rotates and translates the fatigue bar so that the whole gage section can be examined. Using this stage along with backscatter electron imaging, it was possible to find and map the location of all the seeds intersecting the surface. After 250 fatigue cycles and periodically at various other times prior to failure, testing was interrupted on selected samples for re-examination in the SEM. Image analysis was used to measure the cracks emanating from the inclusions. The interrupted testing was limited to the $0.8 \%$ and $0.6 \%$ strain ranges.

After failure, fractography was performed on all specimens to determine the initiation site. Image analysis was performed to determine the size of the initiation flaw.

\section{Powder Cleanliness Quantification Results}

Quantifying cleanliness from a lifing point of view requires the determination of both the density of inclusions, $\mathrm{N}_{\mathrm{V}}$ (no. /unit volume), as well as their size distribution $\left\langle\mathrm{d}_{\max }\right\rangle$. Ultimately, these parameters dictate the occurrence rate, $\mathrm{N}_{\mathrm{A}}$ (no. /unit area), of inclusions on critical surfaces (bores, bolt holes, etc).

$$
\mathrm{N}_{\mathrm{A}}=\mathrm{N}_{\mathrm{V}}<\mathrm{d}_{\max }>
$$

Various techniques have historically been employed to quantify these parameters. Water elutriation was initially utilized with coarse powders. As inclusion levels decreased, electron beam testing and acid dissolution testing was performed in an effort to agglomerate the low fraction of inclusions.

At current levels of inclusion content, fatigue testing, metallography, and heavy liquid separation (HLS) form the basis for the quantification of powder cleanliness. In this study, these methods were evaluated using the seeded material where the number density $\left(\mathrm{N}_{\mathrm{V}}\right)$ and size distributions $\left(<\mathrm{d}_{\max }>\right)$ of the inclusions were well characterized and well controlled.

\section{Heavy Liquid Separation (HLS) Testing}

The HLS testing was performed by the RJLee Group. In this process metal powder is mixed with a heavy liquid whose density is less than that of the Ni powder but greater than the density of the inclusions [5]. The lighter inclusions float in the liquid which is decanted and filtered. The filters are examined using a special automated SEM that can map the filters and uses EDS to identify inclusions. Once the inclusions are identified, image analysis is performed to determine their size.

In the case of the seeded powder, the HLS process was applied to $25 \mathrm{~g}$ powder samples extracted after the seeds were blended with the powder and before the extrusion cans were filled. For the unseeded powder, where inclusions were much rarer, a $250 \mathrm{~g}$ sample was analyzed.

The HLS process was very successful in recovering the seeds from the powder. For the Ram90 $(-270+325)$ seeded material, the HLS process consistently recovered $60 \%$ to $70 \%$ of the expected number of seeds in several trials. For the larger AlcoaT64 (-140+170 mesh) seeded material HLS typically recovered $80 \%$ to $90 \%$ of the expected seed density.

Comparing the size distributions of the recovered seeds to the original input seed distributions, Figure 2, shows remarkable correlation for both seeding types. This suggests that the HLS process is a powerful method for quantifying cleanliness. This also implies that blending does not have any effect on seed size distribution.

The size distribution of natural inclusions recovered from the same unseeded powder was similar to the distribution of the Rem90 seeds recovered by HLS. The Ram90 seeds were recovered by HLS at a rate of $>200$ seeds $/ \mathrm{cm}^{3}$, while the unseeded material the inclusion density was $<0.5$ seeds $/ \mathrm{cm}^{3}$. Based on HLS characterization, the quality of cleanliness of the unseeded powder used in this study compared well to other unseeded -270 mesh production powders from various vendors. The number of seeds recovered by HLS from a $25 \mathrm{~g}$ sample of seeded material ( 180 for Alcoa T64 and $>840$ for Ram90) represents approximately the same number of inclusions expected in the volume of a seeded fatigue bar. The size distribution of the natural inclusions shown in Figure 2 represent 
what is expected in the volume of about 50 unseeded fatigue bars.

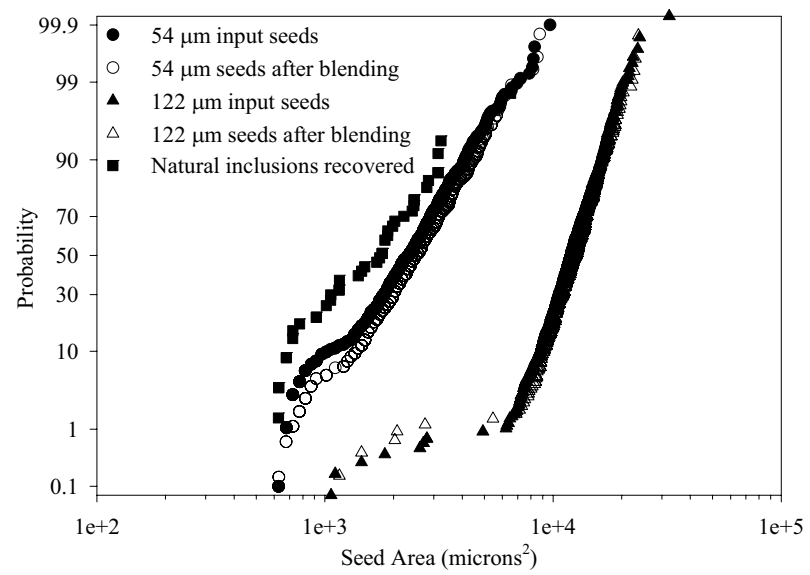

Figure 2. Comparison of size distributions of seeds and natural inclusion recovered using HLS.

Quantitative Metallography

In order to study the effect of mechanical processing on inclusion morphology, extensive quantitative metallography was performed on chord slices taken from extrusions and forgings of both seeded and unseeded material. Image analysis was used to quantify the size distribution of the inclusions. Typical seed morphologies observed in the extrusion and forging are shown in Figure 3. Both processes effectively break up and preferentially orient the seeds. Similar behavior was also observed for the natural inclusions. The seeds, in the forging, tend to lie flat in the axial plane and be preferentially strung out in the radial and tangential orientation. Comparing the size distribution of the seeds from the metallographic sections to the initial input distribution, Figure 4, clearly shows a decrease in seed size after both the extrusion and forging process. This decrease is certainly a manifestation of the break up and orientation of the seeds.
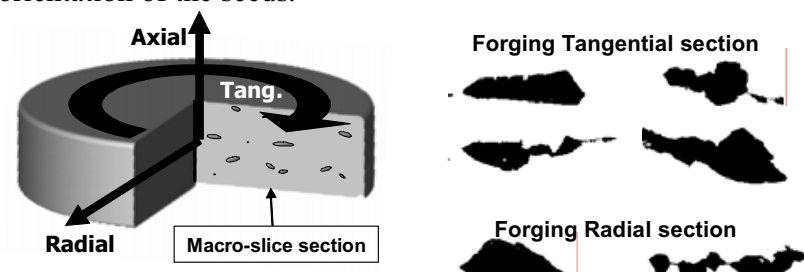

Extrusion Tangential section

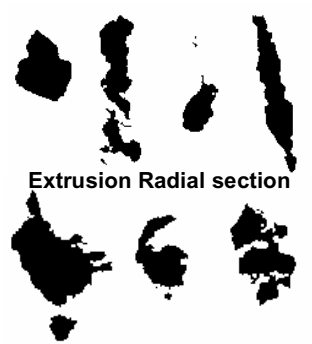

a)
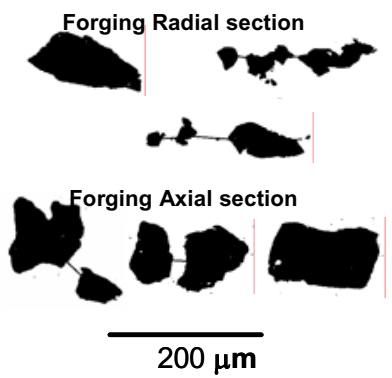

b)

Figure 3. Typical seed morphologies observed for various section in a) extruded material and b) forged material. The processing breaks up and preferentially orients the inclusions.

The resulting seed size distributions taken from tangentially orientated metallographic sections of the various forgings are compared in Figure 5. Metallography also seems capable of quantifying cleanliness given enough surface area is examined. Significant surface area, $3500 \mathrm{~mm}^{2}$ to $10,000 \mathrm{~mm}^{2}$ was examined per forging in order to generate the data shown in Figure 5. Although this suffices to distinguish between the two seeded material, due to the low occurrence rate, significantly more area would be required to accurately differentiate between two unseeded samples.

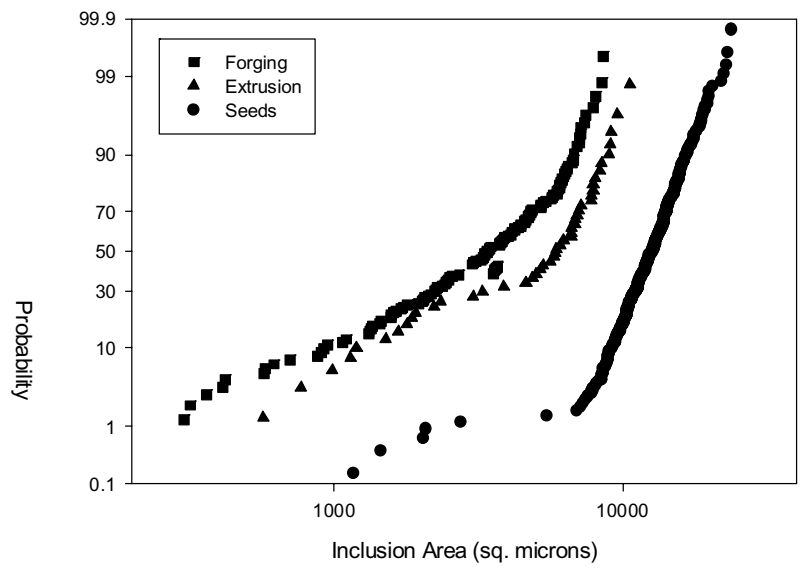

Figure 4. Seed size distributions compared after processing

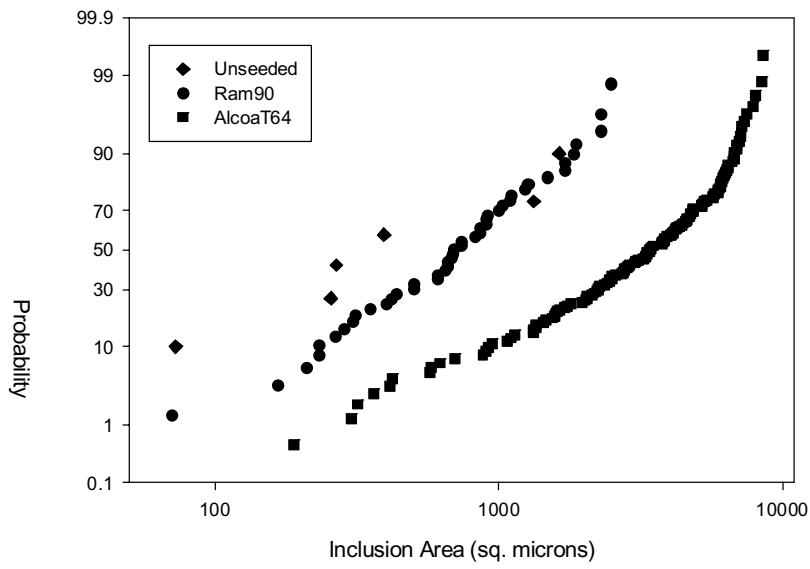

Figure 5. Seed size distributions from tangentially oriented metallographic sections from seeded and unseeded forgings

\section{Low Cycle Fatigue Results}

The effect of powder cleanliness is clearly evident on the fatigue life of the material. The seeds reduced the average fatigue life significantly with the larger AlcoaT64 seeds typically resulting in the lowest lives. As shown in Figure 6, both the size of the inclusion as well as the number of inclusions is needed to properly define cleanliness. The increased occurrence rate in the seeded material has shifted the failure mode from internal to the surface especially for the low strain ranges at $\mathrm{R}=0$ and $\mathrm{R}=0.5$. The failure mode in the unseeded material transitions from 
surface to internal at strain ranges $\Delta \varepsilon=0.8 \%$ and lower. For the unseeded material this transition to internal initiation never occurs for $\mathrm{R}=0$ and $\mathrm{R}=0.5$. At $\mathrm{R}=-1$, in the seeded material the transition occurs at slightly lower strains, $\Delta \varepsilon=0.7 \%$.

In general, for most stress conditions, relatively bigger life debits were observed between the unseeded material and the Ram90 even though they were both nominally screened at -270 mesh. Based on the observation that both the HLS and metallography inclusion size distributions were similar for the seeded Ram90 $(-270+325)$ and the unseeded material $(-270)$, little difference would be expected. However, the increased occurrence rate in the seeded material has shifted the failure mode from internal to the surface resulting in a significant life debit, especially at the low strain ranges for $\mathrm{R}=0$ and $\mathrm{R}=0.5$. Part of the life debit is undoubtedly associated with the fact that at the surface the environment contributes to faster crack propagation.

The effect of inclusion size is more evident for the conditions where the failure modes are consistent. At the lower strain ranges, $\Delta \varepsilon=0.7 \%$. and $\Delta \varepsilon=0.6 \%$. at $\mathrm{R}=-1$ both the seeded and unseeded material fail internally. Under these conditions the life debits due to inclusion size are significantly less, both between the seeded material and the unseeded material. Similarly, at the highest strain ranges where all the material fails from surface inclusions the life debits again are not as significant.
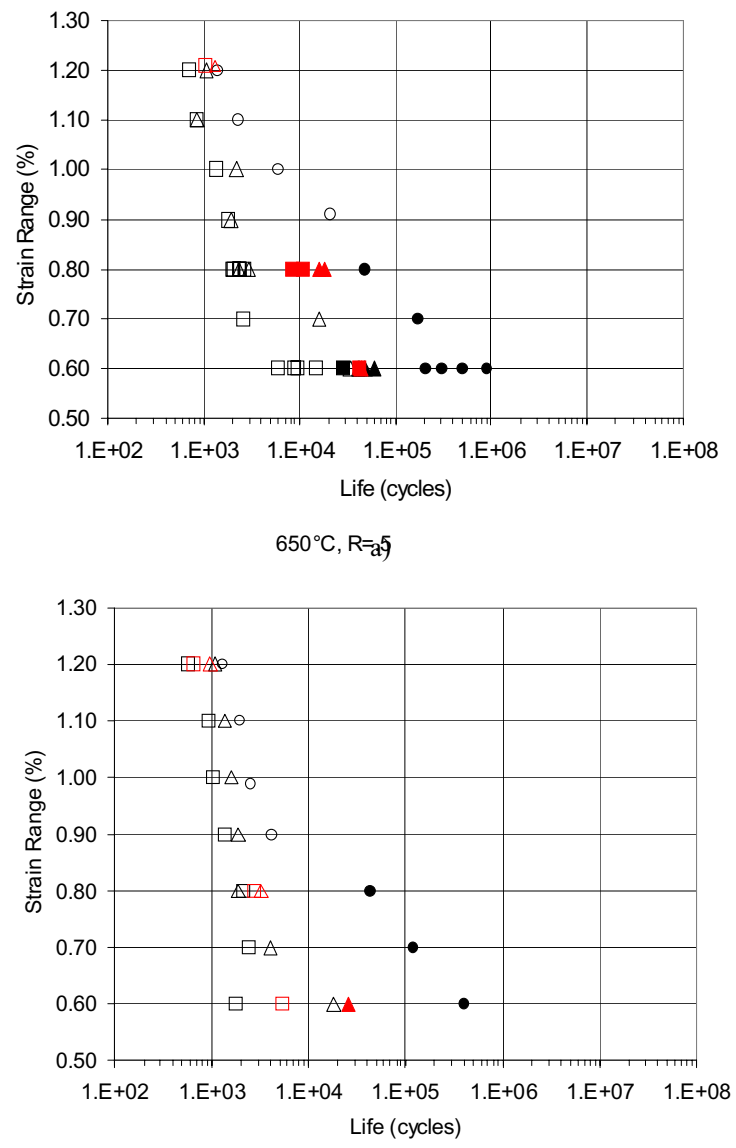

b)
The relative effect of both the size and number of inclusions present is made evident by shot peening. As shown in Figure 6a, at $\mathrm{R}=0$ for the strain ranges of $\Delta \varepsilon=0.6 \%$ and $\Delta \varepsilon=0.8 \%$, shot peening forces the initiation of both seeded material internally. This results in a modest improvement in life. As expected, the life of the T64 seeded material is still significantly less than the unseeded because of the expected differences in the size of the inclusions. The fact that the Ram90 seeded material does not regain similar fatigue capability as the unseeded material reflects that larger flaws are likely to be present when the inclusion rate is significantly higher. This was later validated by fractography.

\section{Inclusion Initiation Behavior Results}

\section{Interrupted fatigue Testing}

Prior to mechanical testing, none of the surface inclusions indicated any cracking even though many of them were broken up due to processing. The interrupted fatigue specimens, which were examined periodically in the SEM, revealed that seeds on the surface caused cracks to initiate very early in life under all fatigue test conditions investigated. A larger fraction of the inclusions monitored initiated cracks at the higher stresses than the lower stresses. Nevertheless, early crack initiation from inclusions was present in all the specimens even in those that ultimately failed from internal initiations.

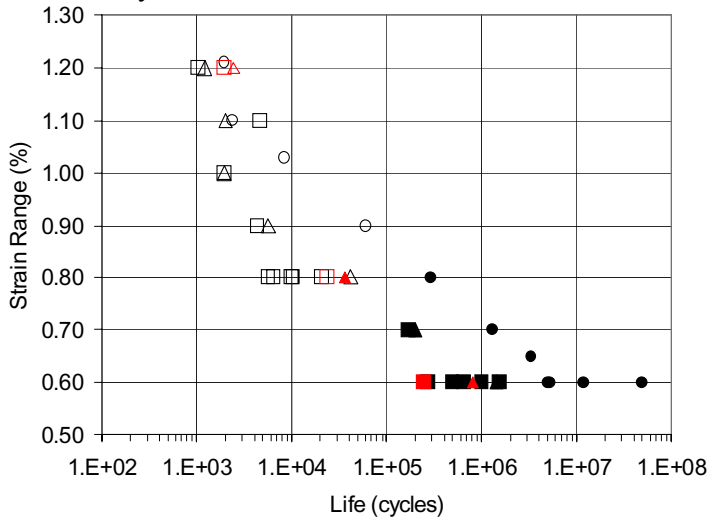

c)

\begin{tabular}{|c|c|c|c|}
\hline \multicolumn{2}{|c|}{ Unpeened } & \multicolumn{2}{|c|}{ Peened (8A/200\%) } \\
\hline 0 & Unseeded & 0 & Unseeded \\
\hline$\triangle$ & $54 \mu \mathrm{m}$ seeded & $\Delta$ & $54 \mu \mathrm{m}$ seeded \\
\hline$\square$ & $122 \mu \mathrm{m}$ seeded & $\square$ & $122 \mu \mathrm{m}$ seeded \\
\hline \multicolumn{4}{|c|}{$\begin{array}{l}\text { Open Symbols depict surface initiation; } \\
\text { Solid symbols depict internal initiation }\end{array}$} \\
\hline
\end{tabular}

Figure 6. LCF lives of unseeded vs. seeded material at $650 \mathrm{C}$ and at a) $\mathrm{R}=0, \mathrm{~b}) \mathrm{R}=0.5$, and $\mathrm{R}=-1.0$ 
Figure 7 shows the crack growth behavior from two typical inclusions on the same specimen as a function of life. After 250 cycles, small "pop-in" cracks can be seen on both inclusions. Although at least on the surface both inclusions seem comparable, in one case the crack propagates to failure, while the other does not propagate further.

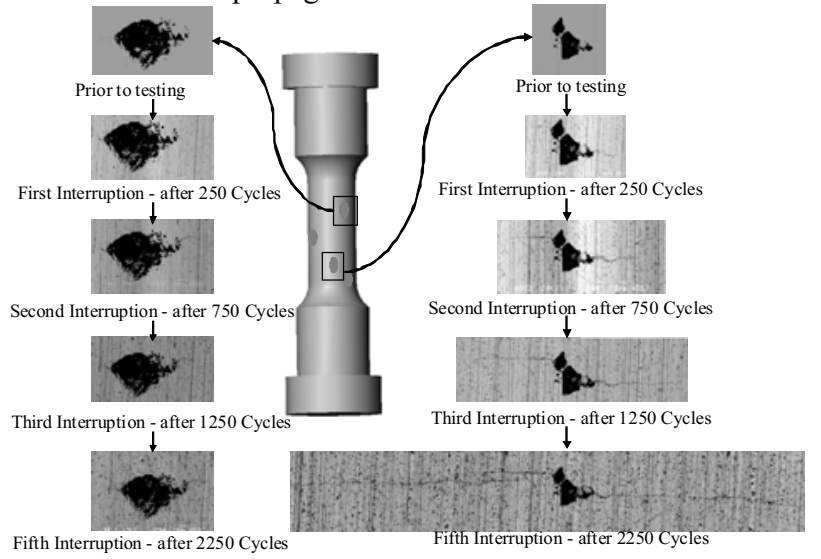

Figure 7. Typical crack growth behavior from surface seeds

These initial cracks may be a result of local residual stress due to coefficient of thermal expansion mismatch, or perhaps, the irregular shaped inclusions generate a very high local stress concentration which in turn is relieved by the formation of the cracks. In this respect, very few surface inclusions display the classical incubation behavior (delayed crack initiation) that has been observed in other seeding studies [6]. Surprisingly enough however, most of the cracks emanating from the inclusions don't propagate or propagate slowly and arrest. This delayed crack propagation for all practical purposes has the same effect as incubation since only a small fraction of the inclusions are capable of causing premature failure.

The relative fraction of active inclusions does vary with test condition, and as expected, at the higher the stress ranges and Rratios more inclusions are active, Figure 8.

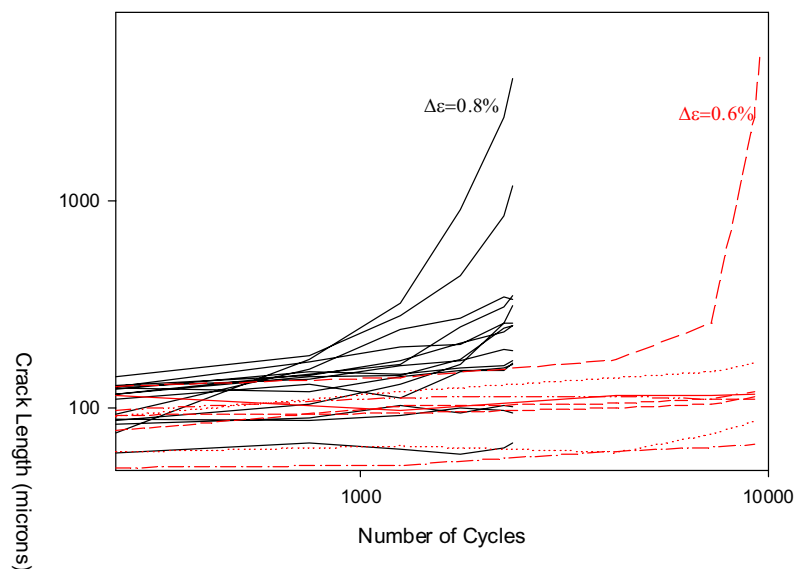

Figure 8. Crack growth behavior of surface inclusions for specimens tested at $650 \mathrm{C}, \mathrm{R}=0$ and $\Delta \varepsilon=0.8 \%$ and $\Delta \varepsilon=0.6 \%$
Fractography

All fatigue bar fracture surfaces were examined after failure and image analysis was performed to quantify the size of the initiating flaw. Typical initiation sites are shown in Figure 9. All seeded bars failed from the seeds regardless of the testing condition. Unseeded bars failed from inherent inclusions as well as other flaws such as porosity and grain facets. On occasion, natural inclusions were found as secondary initiation sites in the Ram90 seeded material. The natural inclusion initiations observed in seeded material were primarily internal. The competition between the natural inclusions and Ram90 seeds at $270+325$ suggests that these seeds behaved realistically.
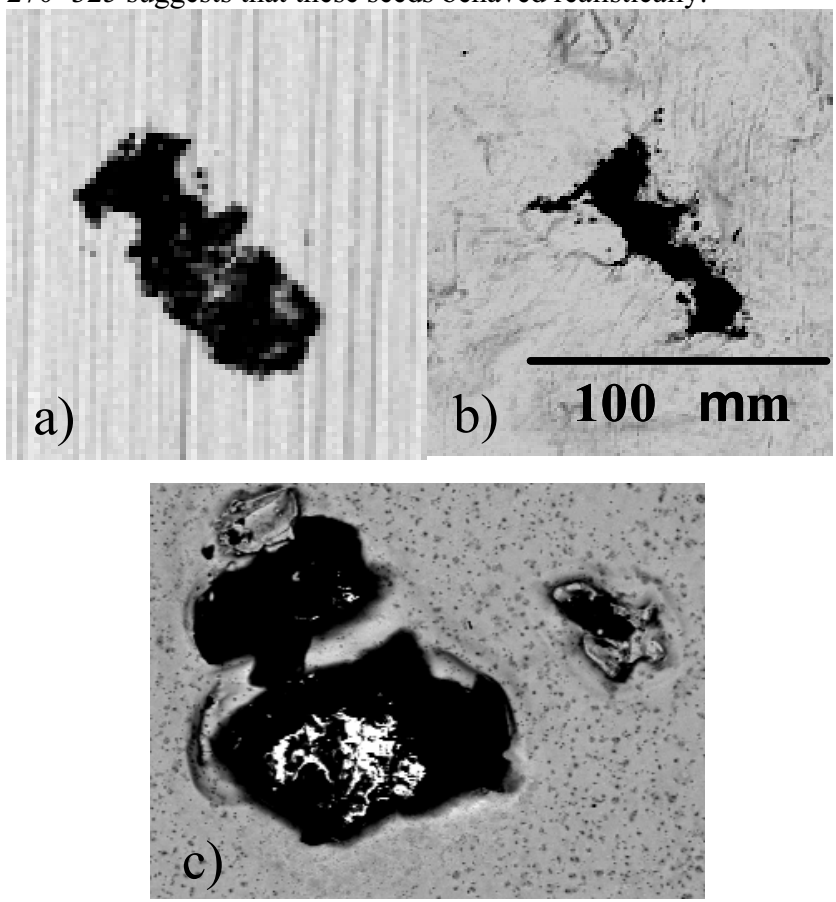

Figure 9. Typical inclusion on LCF bar surfaces; a) asmachined, b) after peening, and c) after electropolishing

In general, many of the inclusions causing initiation were broken and oriented. This confirmed the metallographic observations indicating that extrusion and forging resulted in seed break up. Some of the seeds, AlcoaT64 in particular, also displayed debonding. Surface initiations frequently favored inclusions that were only barely sectioned by the surface and whose bulk was mostly contained within the specimen.

The various size distributions of crack initiating inclusions that caused failure are shown in Figure 10. Internal initiations typically occurred at much larger inclusions than surface initiations. This is to be expected, since the largest flaw is a function of the sampled volume and the volume of material associated with surface connected flaws is small compared to the specimen volume.

Comparison of the size distribution of initiating flaws with the initial size distribution of the seeds from HLS can be seen in Figure 11. The initiating flaw size distribution is larger than the original for both of the seeded material. This observation reflects the fact that fatigue failure is an extreme process. The worst flaw in a given test volume can very well be an unbroken 
unfavorably oriented seed given the fact that so many inclusions are present in a seeded test bar ( 180 for Alcoa T64 and $\sim 840$ for Ram90).

The converse is true for the unseeded material. The initiating flaw size distribution observed in the unseeded material is smaller than the HLS recovered inclusion distribution. This is an indication of the level of cleanliness of the unseeded material. The unseeded material is so clean (inclusions are so rare) that it is unlikely to find a large inclusion in the volume tested even though large inclusions (as large as the Ram90 seeds) are present in the powder. This of course is one reason why seeding studies are cost effective.

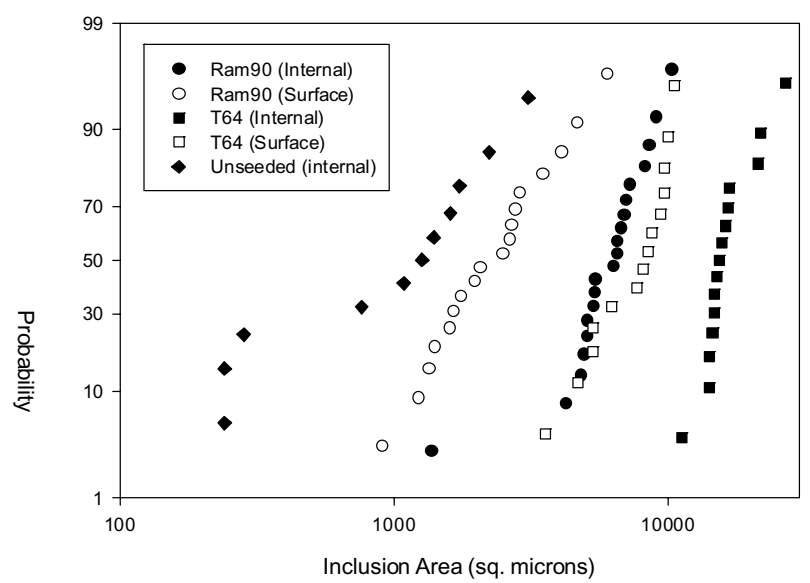

Figure 10. Size distributions of internal and surface flaws of seeded and unseeded material.

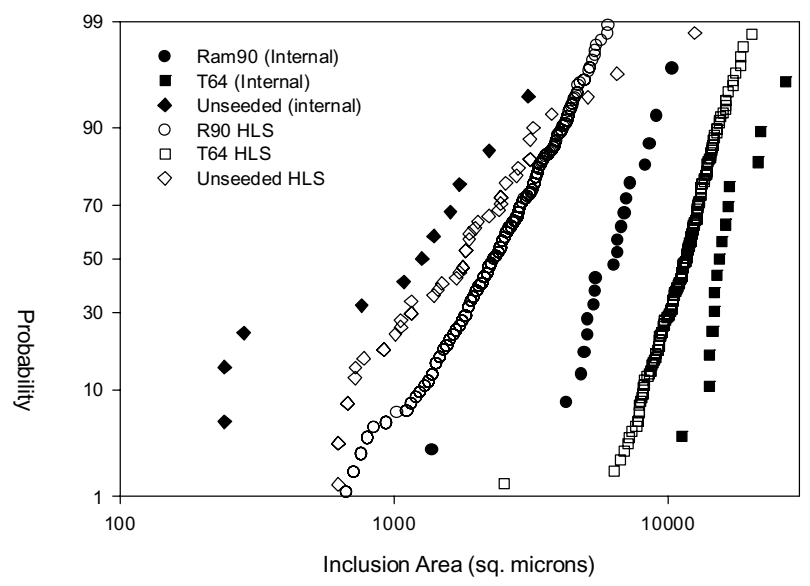

Figure 11. Comparison of the HLS size distributions of seeds and the size distribution of initiating flaws.

\section{Discussion}

\section{Powder Cleanliness}

In this study, three methods were used to evaluate powder cleanliness: HLS, quantitative metallography, and quantitative fractography. All of three methods are useful and capable of quantifying cleanliness to various degrees. A combination of all three methods is desirable in order to incorporate the effects of mechanical processing and to correlate the size and number of inclusions to the resultant fatigue life.
Mechanical processing results in seed break up and preferred orientation and thus should result in a smaller effective inclusion size distribution than that expected from the actual powder mesh. The effect of mechanical processing on the orientation of the seeds is shown in Figure 12. The orientation of the seeds in the forging is not random but strongly aligned with the forging flow. Similarly, the aspect ratio (major length/minor length) of the inclusions in the forging is much greater than the original seeds, Figure 13. This is a manifestation of the breaking and preferred orientation that occurs during processing. As shown by the resulting reductions in area distribution of seeds in the extrusion and forging, (Figure 5), these effects are not negligible. These effects are likely to be even more significant in large forgings were the material flow and deformations are appreciably larger than in the pancake forgings used in this study.

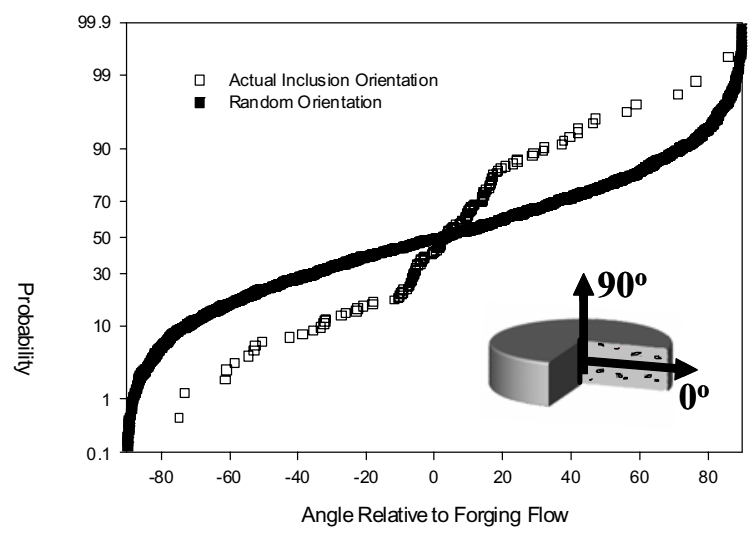

Figure 12. The orientation distribution of seeds observed in the tangential orientation of forging in comparison with the expected random distribution,

Due to the high inclusion rate in seeded material, metallography can be useful in quantifying the effect of processing on inclusion morphology. A main drawback in using metallography as a means for quantifying powder cleanliness is that when dealing with relatively clean powders large surface areas need to be examined in order to statistically quantify the material.

Fatigue testing and quantitative fractography is a powerful method of evaluating cleanliness, since fatigue life is greatly affected by the dirtiness of the powder. The greatest drawback in relying only on fatigue testing is in extending the information to larger volumes.

The fatigue test typically fails from one inclusion, usually the largest in the volume or on the surface, without giving any real indication of how many inclusions are present in the tested volume. The number of inclusions in a given volume is an important parameter, as shown by the seeded fatigue results. The density of inclusions influences both the failure location and the size of the initiating flaw. This was evident when comparing the behavior of unseeded material to the Ram90 seeded material. Both have nominally the same mesh size distribution but significantly different initiating flaw size distributions and as result different fatigue lives.

The fact that mesh size alone is not a good indicator of cleanliness, and that the density of inclusions needs to be accounted for is evident when trying to differentiate between a clean coarse powder (Lot A at -100 mesh) and a finer but dirtier powder (Lot B at -200 mesh). The size distributions of initiating 
flaws are shown in Figure 14 for both powder lots. The cleaner powder, although twice as coarse as the finer powder has slightly smaller initiation flaws. To put it in perspective, both these production quality powders are not significantly dirtier than the intentionally seeded Ram90 material, which was sieved at $-270+325$ mesh.

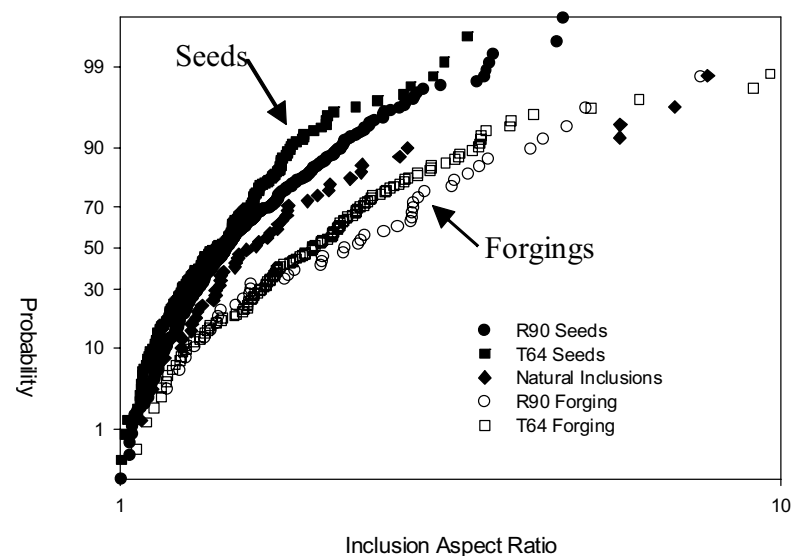

Figure 13. Comparison of inclusion aspect ratio as observed before seeding and after processing in the forging.

The underlying premise that governs this phenomenon is that the more inclusions that are present in a given volume the greater the probability for finding a large inclusion. The same holds true when increasing the stress volume from a fatigue bar to a turbine disk. For a given inclusion density, the bigger the stress volume the greater the probability of finding a large inclusion. This is the main reason that it is important to quantify both the size distribution and inclusion rate. Not knowing the inclusion rate makes more difficult to distinguish between two powder lots; one having a few large inclusions from another having many smaller inclusions. Similarly, it is equally difficult to extrapolate from the extreme flaw size distributions obtained from fatigue bars to those expected in a turbine disk or to a fleet of turbine disks.

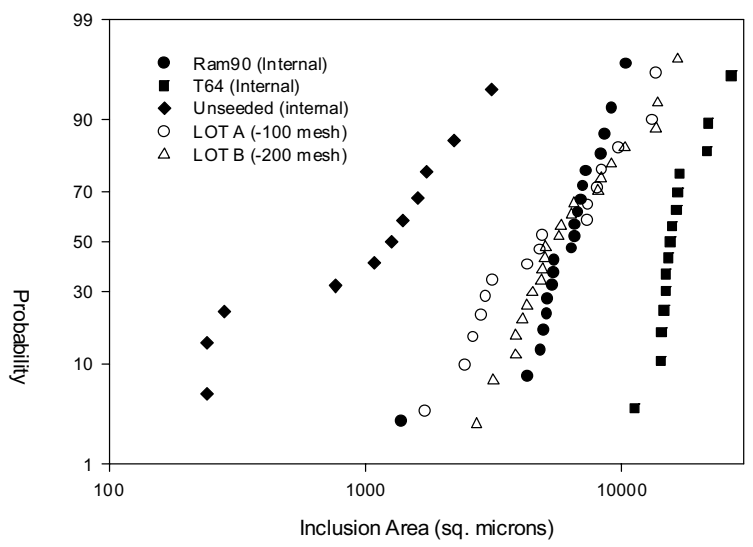

Figure 14. Comparison of initiation flaw size distributions of production powder of various mesh sizes and cleanliness.

Of all the methods evaluated in this study, HLS is the only method that can provide both the size distribution as well as the inclusion occurrence rate. Furthermore, the HLS method proved capable of reliably recovering alumina type inclusions from both seeded and unseeded powder with varying degrees of cleanliness. This method would be also be extremely useful in a production quality control environment since it examines the powder prior to any substantial amount of value added processing has been incurred.

Even though, from a lifing perspective, the HLS analysis provides the necessary information to access cleanliness, disk material typically undergoes additional mechanical processing such as extrusion and forging which ultimately affects the inclusion behavior by breaking up and preferentially orienting the inclusions [7]. The HLS inclusion distributions need to be adjusted to take into consideration the extent of inclusion break up and orientation, after all this should prove beneficial to fatigue life.

Inclusion Modeling

A Monte Carlo simulation of the seeding process, explained in detail elsewhere [8], was developed in conjunction with the HLS, and metallographic, data from the seeded material in this study. At this time, the primary objective of the simulation is to determine the expected distributions of initiating flaws. Taking into consideration: 1) the initial size distribution of inclusions and inclusion density from the HLS results, 2) the orientation of the inclusions from metallography of the final forging, and 3 ) assuming the inclusions were ellipsoids and occurred randomly, the model was able to predict the expected flaw distributions on the surface and internally for the fatigue bars tested, Figure 15.

Success in predicting the flaw distribution size for natural inclusions in the unseeded material implies that given the proper inputs for cleanliness the expected flaw distributions can be accurately determined for any volume and surface area, be it an fatigue bar, a turbine disk, or a fleet of turbine disks.

Once cleanliness has been quantified and the expected flaw distribution can be determined, it is relatively straightforward to apply deterministic linear elastic fracture mechanics (LEFM) to determine life.

Given the early onset of crack initiation from seeds, it was assumed that the experimentally measured initiating flaw sizes might be used as initial flaws to predict the fatigue life. The LEFM based fatigue crack life was determined using AFGROW and assuming no threshold behavior. The results are shown in Figure 16 for surface initiations and Figure 17 for internal initiations.

It is obvious that the larger the inclusion initiation size the lower the resultant fatigue life. This is true for both seeded material as well as the unseeded material; and it is also true for both internal and surface initiations. The predicted LEFM fatigue crack growth lives however, were significantly conservative than those observed experimentally. This is a manifestation of the delayed crack propagation observed in the interrupted testing and suggests that the initial cracks don't behave as sharp fracture mechanics cracks. Surface residual stresses may also probably play an important role since they can slow down cracks. As shown in Figure 16, peened specimens that failed from surface initiations typically showed improved life. Another factor that can attribute to the delayed crack propagation is the intrinsic threshold behavior of small cracks, which was not modeled.

Although the LEFM fatigue crack growth lives serve as a lower bound for predicting the life from the initiating inclusion, it should be noted that for the seeded material there are 
significantly more surface inclusions for which these predictions may be even more conservative.

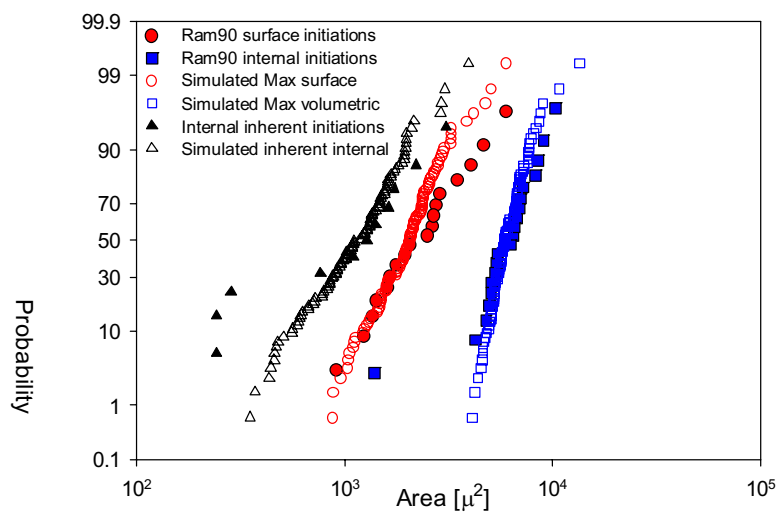

a)

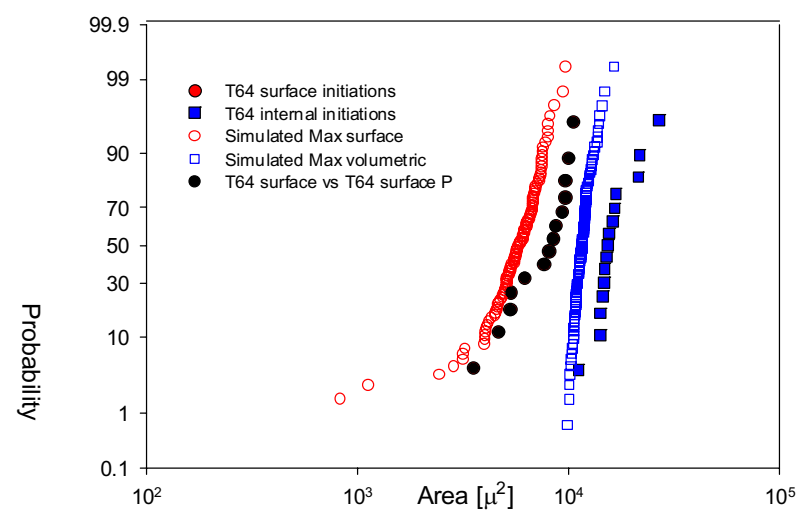

b)

Figure 15. Monte Carlo predictions of initiation flaw size distributions for; a) the unseeded and Ram 90 seeded fatigue bars and b) the Alcoa T64 seeded bars.

Even though inclusions are deleterious to fatigue life, factors such as; 1) their rarity and randomness, 2) their breakup and orientation due to mechanical processing, 3) delayed crack propagation, and 4) surface residual stresses, all help lessen their overall impact on the life of disk components. Extending the analogy of the seeding study to unseeded material would suggest that for stress conditions typical of the turbine disk environment only a small fraction of the surface inclusions encountered would be capable of resulting in premature failure. This behavior can prove to have a big impact on lifing of disk components.

The seeding study suggests that failure from inclusions is probabilistic in nature. In order to take full advantage of this behavior, development of probabilistic models is not only appropriate but necessary. To this end, carefully designed seeding studies can be extremely useful in generating probabilistic data that can be extended to clean powder.

The next step, predicting the fatigue life associated with a given level of cleanliness requires not only an understanding of inclusion initiation behavior but also adequate quantification of these critical parameters.

In the future, further testing and analysis of the data is planned in order to determine the probabilistic nature of incubation and the factors controlling the location of initiation.

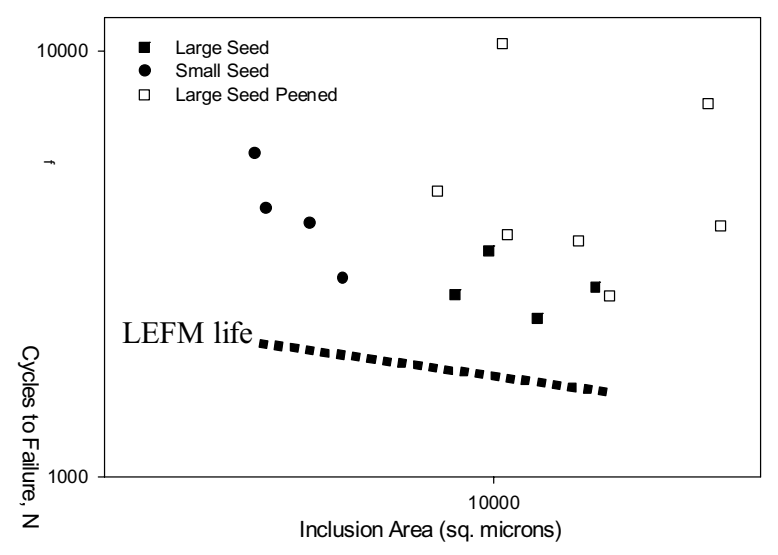

Figure 16. LCF life as a function of initiation flaw size for $\mathrm{R}=0$ and $\Delta \varepsilon=0.8$. All initiations are surface connected.

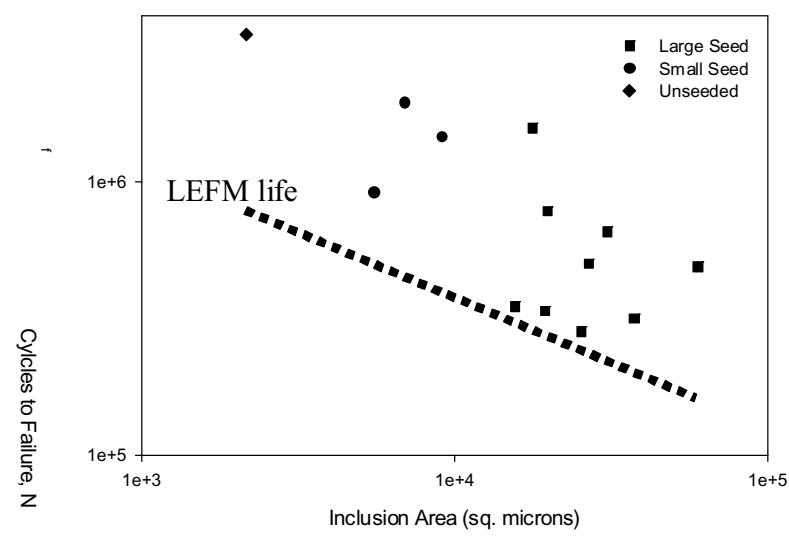

Figure 17. LCF life as a function of initiation flaw size for $\mathrm{R}=-1$ and $\Delta \varepsilon=0.6$. All initiations are internal.

\section{Conclusions}

Well controlled seeding studies prove very useful in studying powder cleanliness and its effect on fatigue life. Due to the high occurrence rate of inclusions they are also a good tool for evaluating the effectiveness of surface modifications such as peening.

Metallography of seeded extrusions and forgings revealed that mechanical processing of powder results in inclusion break up and preferred orientation. Additional work is required to determine and quantify the potential benefit to fatigue life.

Fatigue testing of seeded and unseeded material revealed that both the size and the number of inclusions are required to properly quantify powder cleanliness.

The presence of large number of inclusions, even if their size distribution is relatively small $-270+325$, results in significant debit in fatigue life. The debit is associated with both a transition in failure mode from internal to surface as well as an increase in the initiation flaw size. 
Heavy liquid separation was efficient in recovering alumina type inclusions from both seeded and unseeded powder. It is the only method that can effectively quantify cleanliness since it provides both the size distribution as well as the number of inclusions in a given volume.

Modeling the seeding process by Monte Carlo simulation revealed that given the proper inputs for powder cleanliness the resultant initiation flaw size distribution can be predicted for any volume/surface area.

Although the presence of inclusions is deleterious to fatigue life, interrupted fatigue testing revealed that only a small fraction of the surface inclusions are capable of causing premature failure. Some inclusions don't even initiate cracks. From the rest of the inclusions, cracks propagate at rates well below the expected LEFM predictions, and many ultimately arrest.

Crack incubation and delayed crack propagation from surface inclusions can prove to have a big impact on the life of turbine disks. The development of probabilistic lifing methodologies would be beneficial in taking advantage of this behavior.

\section{References}

1. S. Reichman and D. S. Chang, Powder Metallurgy. Superalloys II, ch. 17, John Wiley \& Sons, New York, NY, 1987, 459-493.

2. T. Banik and K. A. Green, "The Mechanical Property Response of Turbine Disks Produced using Advanced PM Processing Techniques," in Superalloys 2000, ed. by T.M. Pollock, et al., (TMS, Warrendale, PA, 2000), 69-74.

3. T.P. Gabb et al., "Assessment of Low Cycle Fatigue Behavior of Powder Metallurgy Alloy U720," Fatigue and Fracture Mechanics: $31^{\text {st }}$ Volume, ASTM STP 1389, eds. G. R. Halford and J. P. Gallagher (West Conshohocken, PA: American Society for Testing and Materials, 2000), 110-127.

4. Impurities in Engineering Materials, ed. Clyde L. Briant, 3145 .

4. T.P. Gabb et al., "Initial Assessment of the Effects of Nonmetallic Inclusions on Fatigue Life of Powder-MetallurgyProcessed Udimet 720", (NASA TM 2002-211571, NASA Glenn Research Center, 2002).

5. P. Roth et al., "Heavy Liquid Separation: A Reliable Method to Characterize Inclusions in Metal Powder," Characterization, Testing and Quality Control, Advances in Powder Metallurgy and Particulate Materials, 2, (Metal Powder Industries Federation, Princeton, NJ 1994).

6. E. Huron and P. Roth, "The Influence of Inclusions on Low Cycle Fatigue Life in a P/M Nickel-Base Disk Superalloy," in Superalloys 1996, ed. by R. D. Kissinger et al., (TMS, Warrendale, PA, 1996), 359-367.
7. P.T. Kantzos et al., "The Effectiveness of Forging Strain on Ceramic Inclusions in a Disk Superalloy," (Paper Presented at the United Engineering Foundation Conference on Gas Turbine Engines, Copper Mountain, Colorado, 25 September 2002).

8. P. J. Bonacuse et al., "Modeling Ceramic Inclusions in Powder Metallurgy Alloys," Fatigue 2002: Proceedings of the Eighth International Fatigue Congress, vol. 2, ed. A.F. Blom, 1339-1346. 
